\title{
Agents’ Behavior in Market Bubbles: Herding and Information Effects*
}

\author{
Pablo Marcos Prieto, Javier Perote \\ University of Salamanca, Salamanca, Spain
}

\begin{abstract}
This paper explores some behavioral factors that may explain the formation of speculative bubbles in financial markets. The study adopts an experimental approach focused on the agents' behavior when facing a "true” bubble and is incentivized to herd and/or receive information about the market sentiment. For this purpose, a straightforward laboratory experiment that reproduces the dotcom market bubble and asks subjects to forecast asset prices in a true dynamic information scenario. The experiment was conducted in the laboratory of the Faculty of Economics at the University of Salamanca and involved 137 undergraduate students in the degree of economics. The results show that incentives to the herding behavior increase the forecasted volatility and thus contribute to the bubble inflation. Nevertheless, this effect may be offset by giving information to the agents about the expected market trend. Therefore, under herding effects, it is the absence of clear signals about market sentiments what inflates the bubble.
\end{abstract}

Keywords: dotcom bubble, laboratory experiment, behavioral finance, herding, market sentiment, market volatility, random effects model

\section{Introduction}

Speculative bubbles appear when asset prices systematically and abnormally exceed their intrinsic or fundamental values (discounted expected capital flows). Bubble formation tends to happen during an extended period of time but they definitely burst (usually abruptly) causing great losses to the owners of the asset and generating financial crashes and risk contagion, as illustrated by the recent subprime and sovereign debt crises.

The evidence of bubbles arises from the 17th and 18th centuries with the well-known cases of the so-called Dutch Tulipmania (Garber, 1989) and the British South Sea Bubble (Dale, Johnson, \& Tang, 2005), and they have frequently happened in financial markets during the last decades. From the classical economics perspective, the presence of bubbles in financial markets implies the existence of market inefficiencies and the violation of the rational expectations hypothesis (Muth, 1961). Their causes have been argued to lie in psychological aspects of human behavior, such as irrational beliefs, overconfidence, cognitive biases, or

\footnotetext{
${ }^{*}$ Project Supported: Javier Perote acknowledges financial support from the Ministry of Economics and Competitiveness under research project ECO2013-44483-P.

Pablo Marcos Prieto, Ph.D. student, Department of Economics, University of Salamanca, Salamanca, Spain.

Javier Perote, Ph.D., Department of Economics, University of Salamanca, Salamanca, Spain.

Correspondence concerning this article should be addressed to Javier Perote, Universidad de Salamanca, Campus Miguel de Unamuno (Edif. F. E. S.), 37007 Salamanca, Spain.
} 
herding behavior — see Shiller (2000) or Aronson (2011) for comprehensive reviews. Furthermore, the study of the causes of the speculative bubbles has benefited from the impulse of behavioral and experimental economics since Vernon Smith and Daniel Kahneman were awarded with the Nobel Prize in 2002-see e.g., Smith, Shuanek, and Williams (1988), Lei, Noussair, and Plott (2001), Dufwenberg, Lindqvist, and Moore (2005), Bossaerts, Plott, and Zame (2007), and Moinas and Pouget (2013) for experiments on bubbles in financial markets.

This paper adopts the experimental approach to investigate on financial markets' bubble formation by designing a very simple experiment to check how agents behave when they observe the typical behavior of a bubble through different incentives and informational scenarios. Particularly, subjects are requested to forecast S\&P500 index during the formation and burst of the dotcom bubble with past information on price and volatility and either the market trend (subjects' expectations) or certain incentives to herd (introduced in the payoff function). The results show that agents' herding behavior increases market volatility and induces bubble formation but information about market sentiment reduces it, thus contributing to the market efficiency.

The next section explains the experimental setting, the procedures to implement it and the research hypothesis in detail. The rest of the paper proceeds with the analyses of the experimental data in every treatment and discussion of the empirical results. Final remarks are summarized in the last section.

\section{Research Method}

\section{Setting}

The design of experiment focuses on the analysis of the agents' perceptions and behavior on the observation and trading of a real bubble. For this purpose, the dotcom bubble occurred during the late 1990s and its subsequent period was chosen. Particularly, four-monthly data from the S\&P500 index spanning from January 1992 and May 2006, comprising a total of 43 periods were used. Figure 1 depicts the time series and its historical spread (maximum and minimum value).

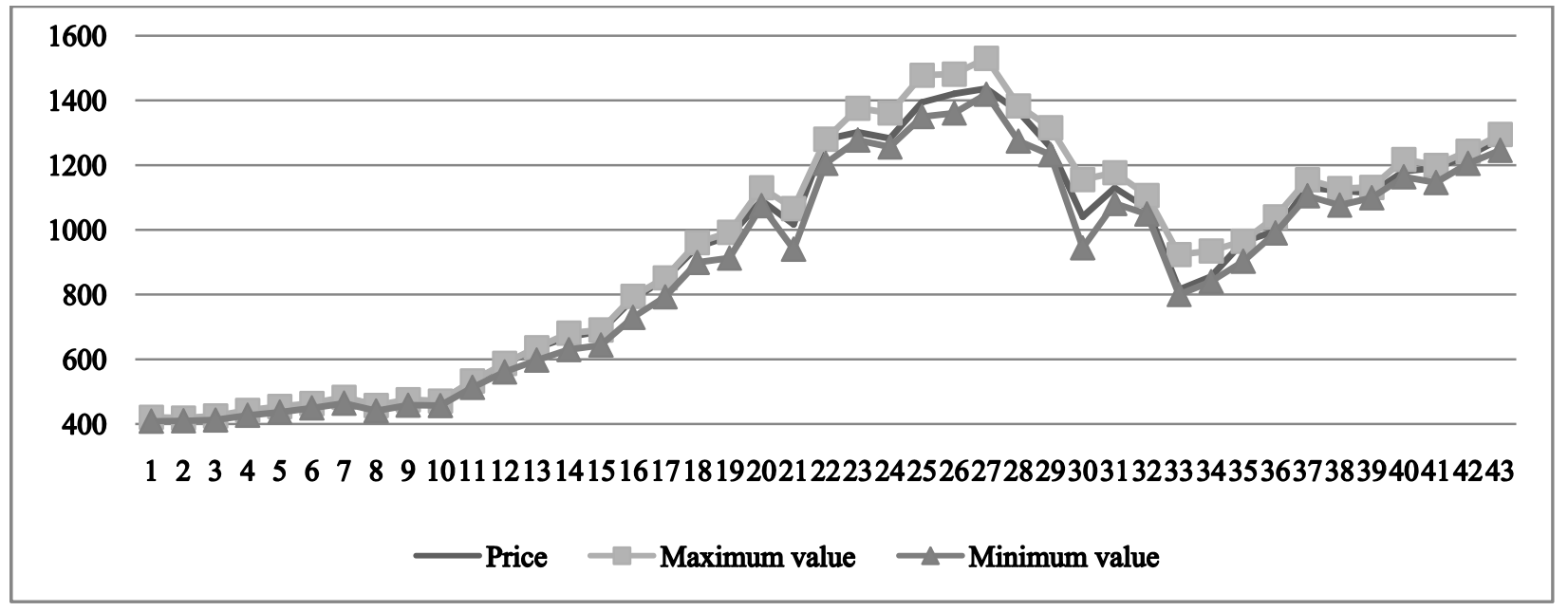

Figure 1. S\&P500 index evolution during the dotcom bubble.

Unlike most of the experiments on bubbles that compute market prices endogenously through the trading decisions of the experimental subjects, the design forces market prices to be exogenous (i.e., the true S\&P500 
series) so that agents' reactions to the available data in every period can be captured by eliciting their one-period-ahead forecasts for asset prices and interpreting their accuracy as a proxy of the gains they would have won on their trading decisions. This frame allows considering an artificial market with controlled prices and observing the agents' decision making in a dynamic information environment as if they were facing a true scenario.

For this purpose, in every period, the experimental subjects receive the information of the past values of the asset price and their corresponding spread, which are dynamically depicted on the computer interface (as shown in Figure 1 for the complete 43 periods of the experiment, i.e. Figure 1 depicts the historical data that subjects observe at the final period of the experiment), and are asked to guess the value of the variable for the next period. This information is updated throughout the experiment and thus subjects know their past forecasting errors every time when they make a new decision (forecast). Agents are incentivized to get more accurate forecasts since their payoffs are inversely related to their forecast errors according to the following function in the baseline or Control Treatment (CT):

$$
\prod_{i t}=100-\left|P_{t}-\hat{P}_{i t}\right|
$$

where $P_{t}$ and $\hat{P}_{i t}$ stand for the market price in period $t$ and its forecast computed by agent $i$, respectively, and thus the absolute value of the forecasted error $\left(P_{t}-\hat{P}_{i t}\right)$ represents a penalty for the subject's under- and over-prediction.

Alternatively, three more treatments are considered. The first one referred to as Payoff Incentives (PI), introduces an additional term in the payoff function that penalizes deviations of the prediction of every subject from the average prediction of all subjects in every period-see equation (2). Such a function incentivizes herding behavior since agents are better off the closer their predictions are to both the true values and the subjects' average predictions.

$$
\Pi_{i t}=100-\frac{1}{2}\left|P_{t}-\hat{P}_{i t}\right|-\frac{1}{2}\left|\hat{P}_{i t}-\hat{P}_{i t}\right|
$$

The second treatment, uses the same payoff function as in equation (2) but delivers new information in the market about Expected Trend (ET), and thus is denoted by PI + ET. For implementing this treatment, in every period, subjects' guesses about the market trend are elicited (with a dummy variable that takes value 1/0 if the subject believes that the price is going to increase/decrease) and publicly report the frequency of subjects that believe that the price is going to increase before they are asked to compute the new forecast.

For the sake of comparison, the experiment includes the third treatment, called as CT + ET, in which the expected trend information is given on the basis of the initially control treatment payoff function-equation (1).

\section{Procedure}

The experiment was conducted in the laboratory of the Faculty of Economics of the University of Salamanca. Subjects were voluntary recruited from the second course of the degree in economics and thus they were supposed to have some skills about macro-economics and financial markets but they had not participated in a laboratory experiment before. A between-subjects design was applied, i.e., different subjects were submitted to only one of the four treatments. The procedure organized two groups of three sessions. In the first 
group of sessions, treatments CT, PI, and PI + ET, with 25, 26, and 23 subjects, respectively, were run and in the second group of sessions, treatments CT, PI, and CT + ET, with 22, 24, and 17 subjects, respectively, were run (a total of 137 subjects participated in the experiment).

Before the experiment had started, instructions were read aloud and experiment did not begin until every subject had understood them. It is worth noting that subjects were not informed about the particular asset and period (i.e., the S\&P500 index during the dotcom bubble) for which they were going to dynamically compute their forecasts, since this information might bias the experimental results. After the experiment, students were privately informed about their performances. The experiment lasted on average approximately 60 minutes.

\section{Hypotheses}

A remarkable feature of bubbles is that market volatility also increases as the asset price increases. This feature is clear from Figure 1 and constitutes one of the main elements on which subjects may build their forecasts. Eventually, one of the main causes of the bubbles is attributed to the fact that agents' trading decisions tend to underestimate volatility by focusing directly on the prices (i.e., expected returns). Hence, and paradoxically, subjects seem to increase market volatility by underestimating it.

In consequence, the study focused on explaining the volatility of the asset price forecasts. Although a priori the introduction of incentives to herding in PI's payoff function should stimulate the convergence of subjects' predictions, on the contrary, it yields to an increase in the volatility of the forecasts (which is consistent with bubble formation). This is because, as they do not have information about the others' beliefs, trying to follow a common trend increases the market noise and its volatility. Nevertheless, if not only "following the herd" is incentivized, but also information on the market sentiment (ET) is delivered, then it is expected that agents achieve convergence on their predictions without the cost of an increasing volatility and bubble inflation. Consequently, the main hypothesis of the study is stated as follows: "Incentives to herding (with) without information about the expected market trend (do not) increase the volatility of the forecasts and (do not) contribute to the bubble inflation”.

\section{Model}

Panel data regression models are considered to explain the standard deviation of subjects' forecasts (Stdprice) as a function of the treatment (and thus comparing alternative treatment cases: CT vs. PI, CT vs. PI + ET, PI vs. PI + ET, CT vs. CT + ET, and PI vs. CT + ET) and a group of control variables. Particularly, the following random effects equation was specified:

$$
\begin{aligned}
& \text { Stdprice }_{i t}=\beta_{0}+\beta_{1} \text { Treatment }_{i t}+\beta_{2} \text { Price }_{i, t-1}+\beta_{3} \text { Spread }_{i, t-1} \\
+ & \beta_{4} \text { Errorprice }_{i, t-1}+\beta_{5} \text { Erroraverage }_{i, t-1}+\beta_{6} \text { Guess }_{i t}+\eta_{i}+\varepsilon_{i, t}
\end{aligned}
$$

where $\eta_{i}$ is a random variable capturing the idiosyncratic effect of subject $i$ and $\varepsilon_{i t}$, the error term for subject $i$ and period $t$ of the model, both satisfy the hypotheses of the random effects regression. The explicative variables of the model are defined below.

Treatment: Dummy variable that takes value 0 for CT and 1 for the other treatments in those comparisons in which CT is involved. PI takes value 0 when comparing this treatment to both PI + ET and CT + ET.

Price: S\&P500 index $\left(P_{t}\right)$; its lagged value captures the information possessed by the subject when forecasting the price for period $t$. 
Spread: Difference of the maximum and minimum value taken by the S\&P500 index; regressions involved lagged values to capture the information available to the subjects.

Errorprice: Forecasted price error in previous period (lagged value) measuring learning effects and adaptive expectations, i.e. $P_{t}-\hat{P}_{i t}$.

Erroraverage: Forecasted price error from the average subjects' prediction in previous period (lagged value), i.e. $\overline{\hat{P}}_{i t}-\hat{P}_{i t}$.

Guess: Dummy variable capturing the subjects' expectations about the market trend, which takes value 1/0 if the subject believes that the price is going to increase/decrease.

\section{Research Results}

\section{Descriptive Statistics}

Table 1 displays some descriptive statistics (mean and standard deviation) for each variable in equation (3) and payoff functions in equations (1) and (2), which stratified for the three different treatments of the first session: CT, PI, and PI + ET.

Table 1

Descriptive Statistics in the First Group of Sessions

\begin{tabular}{lllllll}
\hline & \multicolumn{3}{c}{ CT (1,075 obs.) } & \multicolumn{2}{c}{ PI (1,118 obs.) } & \multicolumn{2}{c}{ PT (989 obs.) } \\
\cline { 2 - 7 } & Mean & Std. Dev. & Mean & Std. Dev. & Mean & Std. Dev. \\
\hline Price & 929.2 & 330.3 & 929.2 & 330.3 & 929.2 & 330.3 \\
Predict & 918.3 & 347.1 & 928.3 & 356.3 & 911.7 & 344.5 \\
Guess & 0.62 & 0.49 & 0.68 & 0.47 & 0.59 & 0.49 \\
Payoff Eq. (1) & 46.1 & 37.2 & 45.2 & 37.7 & 46.4 & 36.6 \\
Payoff Eq. (2) & 54.1 & 33.2 & 51.3 & 35.1 & 54.3 & 32.8 \\
Errorprice & 74.1 & 77.4 & 78.5 & 80.4 & 73.7 & 78.4 \\
Erroraverage & 64.7 & 62.6 & 62.8 & 58.9 & 65.1 & 62.8 \\
Stdprice & 47.9 & 36.9 & 61.1 & 40.8 & 47.6 & 35.9
\end{tabular}

Table 2

Descriptive Statistics in the Second Group of Sessions

\begin{tabular}{|c|c|c|c|c|c|c|}
\hline \multirow{2}{*}{ Variable } & \multicolumn{2}{|c|}{ CT (946 obs.) } & \multicolumn{2}{|c|}{ PI (1,032 obs.) } & \multicolumn{2}{|c|}{ CT + ET (731 obs.) } \\
\hline & Mean & Std. Dev. & Mean & Std. Dev. & Mean & Std. Dev. \\
\hline Price & 929.25 & 330.40 & 929.25 & 330.38 & 929.25 & 330.45 \\
\hline Predict & 912.46 & 348.04 & 909.33 & 351.06 & 926.71 & 348.38 \\
\hline Guess & 0.62 & 0.49 & 0.61 & 0.49 & 0.65 & 0.48 \\
\hline Payoff 1 & 45.60 & 37.22 & 44.36 & 37.38 & 44.35 & 37.49 \\
\hline Payoff 2 & 50.66 & 33.93 & 48.41 & 34.75 & 51.04 & 34.56 \\
\hline Errorprice & 76.73 & 79.33 & 87.04 & 103.65 & 82.64 & 91.55 \\
\hline Erroraverage & 42.34 & 52.47 & 55.10 & 84.50 & 48.77 & 74.72 \\
\hline Stdprice & 50.87 & 29.79 & 77.87 & 58.86 & 45.65 & 33.54 \\
\hline
\end{tabular}

The first observation is that herding function (PI) reduces market's efficiency and subjects' welfare since it significantly increases the prediction error and reduces subject's payoffs-particularly for that in equation (2). 
In fact, average payoff in PI are 2.14\% and 5.28\% lower than that of CT according to equations (1) and (2), respectively. Furthermore, PI increases the optimism about the increasing trend of the market compared to CT according to the averaged guesses of the subjects and this fact has a positive impact on price forecasts and bubble inflation. These findings are also patent in the sharp increase in the volatility of the forecasts.

The second observation is that the information about the expected market trend in PI + ET seems to reduce subjects' optimism (guesses are 8.98\% lower than that of PI), their price forecasts and corresponding errors and thus improve subjects' payoffs. All of these yield to a significant reduction of the volatility and a smoother bubble formation.

The results are also consistent with the descriptive statistics for the second group sessions, with the exception of the fact that subjects' guesses do not significantly increase in PI compared to CT. It is worth noting that new treatment CT + ET also involves a significant reduction of volatility compared to PI, although price forecasts and payoffs do not seem to improve with respect to the other treatments.

\section{Regression Analysis}

Tables 3 and 4 display the random effect estimates of the coefficients and corresponding $t$-ratio statistics of the model in equation (3). One (two) asterisk(s) indicate(s) that the coefficient is significantly different from zero at $10 \%(5 \%)$ confidence level.

Table 3

Models for Forecast Volatility (Stdprice) in First Session of Treatments

\begin{tabular}{|c|c|c|c|c|c|c|}
\hline \multirow{3}{*}{$R$-squared } & \multicolumn{2}{|c|}{ CT vs. PI } & \multicolumn{2}{|c|}{ PI vs. PI + ET } & \multicolumn{2}{|c|}{ CT vs. PI + ET } \\
\hline & \multicolumn{2}{|c|}{0.75} & \multicolumn{2}{|c|}{0.60} & \multicolumn{2}{|c|}{0.66} \\
\hline & Coef. & $t$-ratio & Coef. & $t$-ratio & Coef. & $t$-ratio \\
\hline Treatment & 8.35 & $9.68 * *$ & -10.25 & $-10.04 * *$ & -0.62 & -0.61 \\
\hline Price $(t-1)$ & 0.03 & $15.97 * *$ & 0.03 & $17.60 * *$ & 0.05 & $23.47 * *$ \\
\hline Spread $(t-1)$ & 0.08 & $23.12 * *$ & 0.06 & $19.02 * *$ & 0.02 & $4.61 * *$ \\
\hline Errorprice $(t-1)$ & 0.02 & $1.92 *$ & 0.01 & 0.86 & 0.02 & $1.90 *$ \\
\hline Erroraverage $(t-1)$ & 0.27 & $28.47 * *$ & 0.26 & $21.97 * *$ & 0.22 & $18.71 * *$ \\
\hline Guess & -0.44 & -0.51 & 0.11 & 0.10 & 1.57 & 1.49 \\
\hline Intercept & -14.97 & $-10.22 * *$ & -4.54 & $-2.75 * *$ & -17.88 & $-10.40 * *$ \\
\hline
\end{tabular}

Notes. * Significant at $5 \%$ confidence; ** Signiticant at $1 \%$ conficence.

The estimates are presented for the two group of sessions and different between-treatment comparisons (CT vs. PI, PI vs. PI + ET, and CT vs. PI + ET for the first group of sessions and CT vs. PI, PI vs. CT + ET, and CT vs. CT+ET for the second group of sessions). The main results obtained from the data displayed in Table 3 are those related to the treatment variable. According to this variable, it is clear that PI has a positive impact on volatility compared to CT, i.e., if agents present herding attitudes but there are no clear signals about the market tendency, then a more volatile market and bubble inflation are expected. Nevertheless, if agents have preferences for herding, and information about their beliefs on market trend is released in the market (i.e., the comparison between PI and PI + ET), then a negative effect in volatility and a reduction in the bubble process seem more likely to occur. The final impact of both treatment effects together (CT vs. PI + ET) leads to a mixed insignificant effect. Therefore, the results seem to confirm both the main hypotheses of this study.

The rest of the results of the panel regression models in Table 3 are consistent with the expected subjects' 
behavior. Particularly, all of them are positive and significant with the exception of the subjects' guesses about future movements in the market trend. Therefore, the higher the past prices, volatilities, and errors, the higher the future volatility forecast, which confirms a significant persistence in asset pricing volatility and some kinds of adaptive learning in expectations. Regarding the learning processes in CT vs. PI, the errorprice is the significant variable, but in PI vs. PI + ET, erroraverage becomes a significant one. However, guesses about future price movements are not necessarily correlated with volatility forecast, although this correlation is more significant when information about these guesses is delivered to the market.

Results are replicated in Table 4 for the second session of treatments. The comparison of treatments CT vs. PI confirms the positive effect of herding on market volatility and bubble formation. On the contrary, information about the market trend significantly reduces market volatility even when the agents do not have a herding pattern on their incentives (CT vs. CT + IT). When compared PI and CT + PI, treatment variable is significantly negative since PI tends to increase volatility and CT + IT to reduce it. Regarding the rest of the variables only the significant and negative effect of erroraverage is different to the results in Table 3, reflecting a different type of adaptive learning (noting that in this case the impact of errorprice in volatility is much higher than that in the regressions displayed in Table 3).

Table 4

Models for Forecast Volatility (Stdprice) in Second Session of Treatments

\begin{tabular}{|c|c|c|c|c|c|c|}
\hline \multirow{3}{*}{$R$-squared } & \multicolumn{2}{|c|}{ CT vs. PI } & \multicolumn{2}{|c|}{ PI vs. PI + ET } & \multicolumn{2}{|c|}{ CT vs. CT + ET } \\
\hline & \multicolumn{2}{|c|}{0.28} & \multicolumn{2}{|c|}{0.29} & \multicolumn{2}{|c|}{0.54} \\
\hline & Coef. & $t$-ratio & Coef. & $t$-ratio & Coef. & $t$-ratio \\
\hline Treatment & 25.01 & $12.77 * *$ & -29.52 & $-13.08 * *$ & -3.15 & $-2.94 * *$ \\
\hline Price $(t-1)$ & 0.05 & $14.72 * *$ & 0.06 & $14.68 * *$ & 0.02 & $10.53 * *$ \\
\hline Spread $(t-1)$ & 0.01 & $2.97 * *$ & 0.02 & $3.82 * *$ & 0.08 & $16.42^{* *}$ \\
\hline Errorprice $(t-1)$ & 0.14 & $10.46^{* *}$ & 0.14 & $9.00 * *$ & 0.09 & $10.55^{* *}$ \\
\hline Erroraverage $(t-1)$ & -0.08 & $-4.59 * *$ & -0.09 & $-4.97 * *$ & -0.06 & $-5.60 * *$ \\
\hline Guess & -0.23 & -0.13 & -1.11 & -0.52 & 0.29 & 0.27 \\
\hline Intercept & -8.14 & $-2.58 * *$ & 11.98 & $3.60 * *$ & 1.81 & 1.01 \\
\hline
\end{tabular}

Note. ${ }^{* *}$ Signiticant at $1 \%$ conficence.

\section{Conclusions}

This paper designs an experiment to show how behavioral and experimental economics may help in the understanding of the causes of the speculative bubbles in financial markets. Herding processes are commonly argued to be one of the explanations of such processes, but the current experiment illustrates that even when agents present this behavioral pattern, it is the absence of clear signals about market sentiments and trends that increases market volatility and contributes to the bubble inflation. In other words, the more efficient are the markets, the more information is delivered to the market, the less likely is the bubble formation.

This result is based on an important assumption about the exogeneity of market prices with respect to agents' decisions, which is only sustainable on the basis of competitive markets where agents assume that their decisions do not have a significant impact on prices. This assumption allows constructing a very simple experiment that reproduces the dotcom bubble and analyzes the subjects' reactions to it in a dynamic "true context” where the volatility of agents' forecasts can be used to test the agents' behavior under alternative 
scenarios, particularly those regarding herding incentives and information about the market sentiment.

\section{References}

Aronson, E. (2011). The social animal (11th ed.). New York: Worth/Freeman.

Bossaerts, P., Plott, C., \& Zame, W. (2007). Prices and portfolio choices in financial markets: Theory, econometrics and experiments. Econometrica, 75, 993-1038.

Dale, R. S., Johnson, J. E. V., \& Tang, L. (2005). Financial markets can go mad: Evidence of irrational behaviour during the South Sea Bubble. The Economic History Review, 58, 233-271.

Dufwenberg, M., Lindqvist, T., \& Moore, E. (2005). Bubbles and experience: An experiment. The American Economic Review, 95, 1731-1737.

Garber, P. M. (1989). Tulipmania. The Journal of Political Economy, 97, 535-560.

Lei, V., Noussair, C. L., \& Plott, C. R. (2001). Nonspeculative bubbles in experimental asset markets: Lack of common knowledge of rationality vs. actual irrationality. Econometrica, 69, 831-859.

Moinas, S., \& Pouget, S. (2013). The bubble game: An experimental study of speculation. Econometrica, 81, 1507-1539.

Muth, J. F. (1961). Rational expectations and the theory of price movements. Econometrica, 29, 315-335.

Shiller, R. J. (2000). Irrational exuberance. New Jersey: Princeton University Press.

Smith, V. L., Shuanek, G., \& Williams, A. (1988). Bubbles, crashes and endogenous expectations in experimental spot asset markets. Econometrica, 56, 1119-1151. 